\title{
INTERVAL METHODS OF ASSIGNING THE NOMINAL TOLERANCES AND CHOOSING ELEMENTS
}

\author{
Galina Shilo $^{1)}$, Nikolay Gaponenko ${ }^{2)}$ \\ 1) Ph.D, Zaporizhzhia National Technical University, Zhukovsky str. 64, Ukraine, 69063 \\ 2) Ph.D student, Zaporizhzhia National Technical University, Zhukovsky str.64, Ukraine, 69063, gshilo@zntu.edu.ua

\begin{abstract}
The procedure of assigning the nominal tolerances is offered. The interval-structure models are used. The influence of exposures is taken into account. The maximum relative volume of tolerances is ensured. The possibility of selecting elements is taken into account.
\end{abstract}

Keywords: - interval methods, interval models, nominal tolerances, compensating the external action

\section{INTRODUCTION}

The task of providing the necessary accuracy of output characteristics is one of the main problems being solved when designing and producing electronic devices. The means of toleranceparameter synthesis for non-linear output functions are practically not available in the modern CAD systems of electronic devices. The problem of creating the software for assigning tolerances, in these cases, can be solved using interval mathematics [1-3].

The method of shaping simplified interval models for non-linear output functions of electronic devices is offered in [4]. Such models allow bringing about the optimization of operational tolerances for parameters of elements of electronic devices taking into consideration the influence of external actions [5]. The interval methods of determining nominal tolerances, which are ensured in producing elements have not been developed by now.

The purpose of the paper considered is the development of methods of assigning the nominal tolerances using the simplified interval models and taking into account the changes of parameters of elements under the exposures. The simplified interval model of non-linear output function of electronic devices takes usually the form [4]:

$$
\mathbf{y}=\mathbf{a}_{0}+\sum_{\substack{i=1 \\ a_{i}>0}}^{n} \mathbf{a}_{i} \mathbf{x}_{i}+\sum_{\substack{i=1 \\ a_{i}<0}}^{n} \operatorname{dual}\left(\mathbf{a}_{i} \mathbf{x}_{i}\right),
$$

where $\mathbf{y}=[\underline{y} ; \bar{y}] ; \quad \mathbf{a}_{i}=\left[\underline{a}_{i} ; \bar{a}_{i}\right] ; \quad \mathbf{x}_{i}=\left[\underline{x}_{i} ; \bar{x}_{i}\right]$ are the interval values of output function, interpolation coefficients and input parameters; dual $\mathbf{x}_{i}=\left[\bar{x}_{i} ; \underline{x}_{i}\right]$ is a dual interval; $n$ is the number of parameters.

The exposures (temperature and humidity changes, aging etc.) result in altering the position of the bounds of intervals $\mathbf{y}$ and $\mathbf{x}$ and consequently interval model (1) is transformed into the expression

$$
\mathbf{F b}(\mathbf{y})=\mathbf{a}_{0}+\sum_{\substack{i=1 \\ \mathbf{a}_{i}>0}}^{n} \underset{\mathbf{a}_{i}}{\mathbf{F b}} \mathbf{F b}\left(\mathbf{x}_{i}\right)+\sum_{\substack{i=1 \\ \mathbf{a}_{i}<0}}^{n} \operatorname{dual}\left(\underline{\mathbf{a}}_{i} \mathbf{F b}\left(\mathbf{x}_{i}\right)\right)
$$

where $\mathbf{F b}(\mathbf{y})=\left(\left[\underline{y}_{m} ; \underline{y}_{p}\right],\left[\bar{y}_{m} ; \bar{y}_{p}\right]\right)$,

$\mathbf{F b}(\mathbf{x})=\left(\left[\underline{x}_{m} ; \underline{x}_{p}\right],\left[\bar{x}_{m} ; \bar{x}_{p}\right]\right)$ are interval structures having floating bounds [6];

$y_{m}, y_{p}, x_{m}$ and $x_{p}$ are the interval bounds for lower and upper values of the external action coefficients.

Mapping the interval-structure equation (2) into the area of interval numbers results in two equations:

$\underline{\mathbf{y}}=\underline{a}_{0}+\sum_{\substack{i=1 \\ \underline{a}_{i}>0}}^{n} \underline{a}_{i} \underline{\mathbf{x}}_{i}+\sum_{\substack{i=1 \\ \bar{a}_{i}<0}}^{n} \bar{a}_{i} \overline{\mathbf{x}}_{i} ; \overline{\mathbf{y}}=\bar{a}_{0}+\sum_{\substack{i=1 \\ a_{i}>0}}^{n} \bar{a}_{i} \overline{\mathbf{x}}_{i}+\sum_{\substack{i=1 \\ \underline{a}_{i}}}^{n} \underline{a}_{i} \underline{\mathbf{x}}_{i}$

where $\left.\quad \underline{\mathbf{y}}=\underline{y}_{m} ; \underline{y}_{p}\right\rfloor ; \quad \overline{\mathbf{y}}=\left[\bar{y}_{m} ; \bar{y}_{p}\right] ; \quad \underline{\mathbf{x}}=\left\lfloor\underline{x}_{m} ; \underline{x}_{p}\right\rfloor ;$ $\overline{\mathbf{x}}=\left[\bar{x}_{m} ; \bar{x}_{p}\right]$.

The bound of interval parameters $\mathbf{x}_{i}$ in Eq. (3) have non-linear dependence on the external actions. These non-linear relations can be described by the simplified interval models: 


$$
\underline{\mathbf{x}}=\underline{x}_{n}\left(1+\boldsymbol{\sigma}_{x} \mathbf{d}\right) ; \overline{\mathbf{x}}=\bar{x}_{n}\left(1+\boldsymbol{\sigma}_{x} \mathbf{d}\right)
$$

where $\boldsymbol{\sigma}_{x}=\left\lfloor\alpha_{x m} ; \alpha_{x p}\right\rfloor$ is the reduced interval coefficient of external actions;

$\mathbf{d}=\left\lfloor d_{m} ; d_{p}\right\rfloor$ is the interval of external actions;

$\underline{x}_{n}$ and $\bar{x}_{n}$ are the lower and upper nominal values

of the input parameters, defined in standard conditions of the environment.

The procedure of assigning the nominal tolerances depends on the degree of knowledge of properties of radio-electronic elements or materials. It is possible to select two main kinds of procedures of assigning of the nominal tolerances:

- The nomenclature of elements and materials has been determined;

- There is a possibility of choosing elements from the data base.

\section{ASSIGNING TOLERANCES WHEN THE NOMENCLATURE OF ELEMENTS HAS BEEN PRESET}

It is assumed that the external action coefficients are determined, and there is the possibility of choice the nominal deviation of parameters if the nomenclature of elements has been given. It is true, for example, for resistors, because the latter are produced of the same materials but at different accuracy. After the external action coefficients have been defined, the endpoint deviations of element parameters are written as

$$
\begin{array}{ll}
\underline{x}_{m}=\underline{x}_{n}\left(1+\alpha_{x m} d_{m}\right) ; & \underline{x}_{p}=\underline{x}_{n}\left(1+\alpha_{x p} d_{p}\right) \\
\bar{x}_{m}=\bar{x}_{n}\left(1+\alpha_{x m} d_{m}\right), & \bar{x}_{p}=\bar{x}_{n}\left(1+\alpha_{x p} d_{p}\right) .
\end{array}
$$

In operational mode, the endpoints of parameters can take any of values (5) or (6). But if the external action coefficients have the same signs, the lower deviations of parameters are evaluated by formulae (5) and the upper ones - by (6). When the values $\alpha_{x}$ are positive ones, the nominal endpoints of parameters are determined by

$$
\underline{x}_{n}=\frac{\underline{x}_{e}}{1+\alpha_{x m} d_{m}} ; \bar{x}_{n}=\frac{\bar{x}_{e}}{1+\alpha_{x p} d_{p}},\left(\alpha_{x m}>0, \alpha_{x p}>0\right)(7)
$$

where $\underline{x}_{e}, \bar{x}_{e}$ are operational bounds of parameters.

If the external action coefficients are negative numbers the nominal bounds are defined by $\underline{x}_{n}=\frac{\underline{x}_{e}}{1+\alpha_{x p} d_{p}} ; \bar{x}_{n}=\frac{\bar{x}_{e}}{1+\alpha_{x m} d_{m}},\left(\alpha_{x m}<0, \alpha_{x p}<0\right)$.

The nominal input parameter bounds obtained allow defining the nominal bounds of the output characteristic and the coefficient of external actions. For this purpose, Eq.(3) is mapped into the area of real numbers and reduced to the system of four equations solved relatively to the nominal endpoints of output parameters and the external action coefficient

$$
\begin{gathered}
y_{n}=\underline{a}_{0}+\sum_{\substack{i=1 \\
a_{i}>0}}^{n} \underline{a}_{i} x_{n i}+\sum_{\substack{i=1 \\
a_{i}<0}}^{n} \bar{a}_{i} x_{n i} ; \\
y_{n}=\bar{a}_{0}+\sum_{\substack{i=1 \\
a_{i}>0}}^{n} \bar{a}_{i} x_{n i}+\sum_{\substack{i=1 \\
a_{i}<0}}^{n} \underline{a}_{i} x_{n i}+; \\
\alpha_{y_{m}} y_{n}=\sum_{\substack{i=1 \\
\underline{a}_{i}>0}}^{n} \underline{a}_{i} x_{n i} \alpha_{x_{i n}}+\sum_{\substack{i=1 \\
a_{i}<0}}^{n} \bar{a}_{i} x_{n i} \alpha_{x_{i n}} ; \\
\alpha_{y_{p}} y_{n}=\sum_{\substack{i=1 \\
\underline{a}_{i}>0}}^{n} \underline{a}_{i} x_{n i} \alpha_{x_{i p}}+\sum_{\substack{i=1 \\
a_{i}<0}}^{n} \bar{a}_{i} x_{n i} \alpha_{x_{i p}} ;
\end{gathered}
$$

This calculations requires in the first line to determining operational tolerances. This procedure is considered in [5].

\section{CHOOSING THE RADIOELEMENTS}

If nomenclature of radioelements and materials is not given the possibility of choosing elements appears. It allows to:

- choose the most maximal external action coefficient;

- expand tolerances of nominal parameters of radioelements.

Thus, the requirements to element accuracy and equipment cost are reduced. Selecting elements and materials must be carried out taking into account their available nomenclature. It is preset by limitations:

$$
\alpha_{x i \min } \leq \alpha_{x i} \leq \alpha_{x i \max },
$$

where $\alpha_{x i \min }$ and $\alpha_{x i \max }$ are minimum and maximum values of external action coefficients.

The set with the maximal leakage field of elements $\alpha_{i}$ is assumed to be the optimal solution of problem of selecting external action coefficients. In this case, to choose the optimal elements the multiplicative criterion (which can be called the 
volume of hyperparallelepiped of external action coefficients) is used.

DEFINITION. The value

$$
S=a x_{n} \alpha_{x}
$$

is called factor of compensation.

The compensation factor can has positive or negative value depending on signs of coefficients $a$ and $\alpha_{x}$. The nominal value of parameters and bounds of coefficients $a$ are used to define the endpoints of compensation factor.

The application of expression (14) allows to write equations (11) and (12) in the form:

$$
\sum_{i=1}^{p} S_{i}^{+}+\sum_{i=1}^{m} S_{i}^{-}=S_{y}
$$

where $S_{i}^{+}$and $S_{i}^{-}$are positive and negative factors of compensation;

$p$ and $m$ are the number of elements having respective factors;

$S_{y}=y_{r} \alpha_{y}$ is the compensation factor of output function.

Taking into account the designation in (14), the system of limitations (13) assumes the form:

$$
S_{i \min } \leq S_{i} \leq S_{i \max }
$$

THEOREM (compensation factors). Let the compensation factor of output function, the number of elements having positive and negative value be preset. The maximum volume of the factors of compensating the elements is reached if the conditions:

$$
\begin{gathered}
p S^{+}+m S^{-}=S_{y}, \\
\left(p \geq 1 \quad \text { if } \quad S_{y}>0 ; \quad m \geq 1 \quad \text { if } \quad S_{y}<0\right)
\end{gathered}
$$

are satisfied.

The theorem is proved by constructing the hyperparallelepiped of maximum volume at the coordinate origin and at the point of compensation hyperplane (15).

COROLLARY 1. The full compensation of external action influence on the output function is reached when

$$
p S^{+}+m S^{-}=0, \quad(p \geq 1, \quad m \geq 1) .
$$

COROLLARY 2. The distinction of sign at least of one factor of compensating elements is the requirement for compensating the of external action influence.

Expressions (17) and (18) allow to organizing the procedure of selecting elements. At the first procedure step, the nominal deviation of output function is preset and the nominal deviation of elements is defined. The algorithm of this step is presented in [5].

Further, the endpoints of compensation factors are chosen taking into account conditions (16):

$$
p S_{0}^{+}=\min \left\{\delta_{i \max }^{+}\right\} ; \quad p S_{0}^{-}=\max \left\{\delta_{i \min }^{-}\right\} .
$$

If the condition

$$
p S_{0}^{+}>S_{y}-m S_{0}^{-}
$$

is satisfied, the value $S_{0}^{+}$is the limiting factor and

$$
S^{+}=S_{0}^{+}, \quad S^{-}=\frac{1}{m}\left(S_{y}-p S_{0}^{+}\right)
$$

are assumed.

The modification of the sign of inequality (19) leads to expressions:

$$
S^{+}=\frac{1}{p}\left(S_{y}-m S_{0}^{-}\right), \quad S^{-}=S_{0}^{-} .
$$

The possibility of obtaining the given level of compensation is defined by conditions

$$
S^{-}<\min \left\{S_{\max }^{-}\right\}, \quad S^{+}>\max \left\{S_{\min }^{+}\right\} .
$$

If these conditions are not satisfied, then it is necessary to change the level of factor $S_{y}$ and to repeat the selecting of elements. At the end of optimization procedure, the external action coefficients are defined by expression (14).

The optimization procedure suggested allows selecting parameters of elements, providing the complete independence of output characteristics from the external actions. This is possible if the nomenclature of elements is wide. In case the nomenclature is limited, it is necessary to increase the coefficients $\alpha_{y}$ to the values ensuring the compromise between the stability of output characteristics and that of element.

\section{CONCLUSIONS}

The interval methods of calculating the nominal tolerances proposed allow carrying out their optimization taking into account the limitations for the parameters of elements or materials. Achieving, 
in this case, the maximum volume of external action coefficients, the requirements to the parameters of elements and the equipment cost are reduced. The advantage of method offered consists in minimizing the influence of exposures on the output characteristics, which approaches the nominal tolerances to the maximally allowable deviations in the operational process.

The interval method of assigning the nominal tolerances may be employed under the conditions of non-linearity of output functions and in the smallbatch production when distributive law of stochastic variable is unknown. These methods may be used in the cases when it is necessary to provide the interchangeability of elements or if they are in small numbers. The statistical approach to calculating the nominal tolerances is preferred in other cases.

\section{REFERENCE}

[1] E. Kaucher. Interval Analysis in the Extended Interval Space IR, Computing Suppl, No. 2 (1980). pp. 65-79.

[2] A. P. Voschynin, G. R. Sotirov. Optimization in vague conditions. MEI. Moscow, 1989. p. 224. (in Russian).

[3] S. P. Shary. Algebraic Approach to the Interval Linear Static Identification, Tolerance and Control Problems, or One More Application of Kaucher Arithmetic, Reliable Computing 2 (1) (1996). pp. 3-33.

[4] G. M. Shylo. Forming of interval models for admittances computation, Radio Electronics. Informatics. Management, No. 1 (2002). pp. 9095. (in Ukrainian).

[5] V. Krischuk, G. Shilo, N. Gaponenko. Optimization of ISLAE solutions in the problems of assigning tolerances for parameters of electronic devices. Proceedings of the International Conference "Modern problems of radio engineering, telecommunications and computer science", Lviv, Ukraine 2002, pp. 114-115.

[6] G. M. Shylo. Intervals and interval structures, Radio Electronics. Informatics. Management, No. 2 (2001). pp. 121-125. (in Ukrainian.)

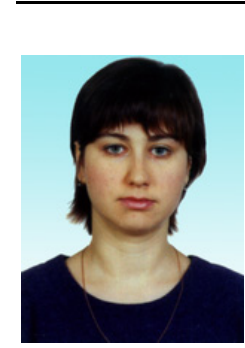

Galina Shilo was born 30 March 1976.

Title: Ph.D., Assistant Professor of Institute of Radio Electronica and Informatics.

Education:

Zaporizhzhya

State Technical University;

1993-1998 software engineer.

1998-2001 Zaporizhzhya National Technical University, Ph.D. student.

2003 Ph.D.

Experience: Sientific work on working up methods of calculating tolerances of electronic device parameters using interval mathematics.

2001- present Zaporizhzhya National Technical University, Assistant Professor of Institute of Radio Electronics and Informatics.

Areas of interests: interval mathematics, CAD systems.

Nikolay Gaponenko was born 24 March 1940.

Title:

Professor.

Education: Taganrog Institute

of Radio Engineering;

1958-1963 radio engineer.

1968-1971 Taganrog Institute

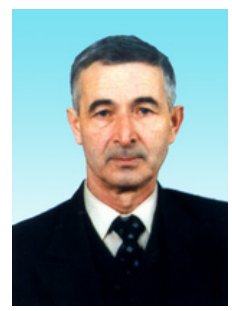

of Radio Engineering; Ph.D. student.

$1971 \quad$ Ph.D.

Experience: Scientific work on working up methods of calculating thermal processes in electronic devices.

Professor's work on theoretical principles of calculatingand designing electronic devices, operations research.

1971-1982 Taganrog Institute of Radio Engineering; Assistant Professor.

1983-1986 Zaporizhzhya Industrial Academy; Assistant Professor.

1986-present Zaporizhzhya National Technical University; Assistant Professor of Institute of Radio electronics and Informatics.

Areas of interests: interval mathematics, radio electronic device design. 